\title{
Transfer after pattern discrimination in primates*
}

\author{
DONALD ROBBINS $\dagger$ \\ Emory University, Atlanta, Ga. 30322
}

Two squirrel monkeys and one infant orangutan were trained on a simultaneous discrimination between two stimuli that were identical except for a 90-deg difference in orientation. Subsequent transfer tests failed to reveal reliably the positive and negative anomalous transfer effects, i.e., preference for a solid-line stimulus over the original positive stimulus in the same orientation and preference for the original negative stimulus over a solid-line stimulus in the same orientation, respectively. Different transfer test procedures were implicated as being responsible for this failure, and it was suggested that additional exploration of the various transfer test procedures should be pursued before the generality of the anomalous effects could be established.

Dodwell (1970) and Dodwell, Litner, \& Niemi (1970) recently replicated and extended the "anomalous transfer effect" first reported by Krechevsky (1938). Dodwell (1970) used hooded rats and squirrels and taught these Ss a simultaneous discrimination between the stimuli shown in Fig. 1A. The training stimuli were four dashed lines in either a horizontal or vertical orientation, which he referred to as "rows" and "columns," respectively. During subsequent transfer tests, Dodwell (1970) and Dodwell et al (1970) found a preference for four solid lines in the same orientation as the positive training stimulus over the positive training stimulus itself (see Fig. 1B for an example). Further, they found a preference for the original negative training stimulus over four solid lines in the same orientation. These results are referred to as positive and negative anomalous transfer effects, respectively. In addition, when both stimuli were solid lines, Dodwell (1970) found a preference for the stimulus in the same orientation as the original positive training stimulus. Finally, when the original positive stimulus was shown along with a stimulus that had solid lines in the same orientation as the original negative stimulus, the original positive stimulus was preferred (Dodwell, 1970). These results were taken as additional evidence that the anomalous effects are not simply a novelty effect.

Dodwell points out that the anomalous transfer effects present difficulties for most extant theories of discrimination and generalization. He concluded that

*This research was supporied in part by a grant from Emory University and in part by Grant RR-00165 from the National Institutes of Health to the Yerkes Regional Primate Center. I wish to express my appreciation to the staff of tine Yerkes Regional Primate Center, where the research was conducted, to David Bachman, who ran the squirrel monkeys, and to Carol $T$. Bush, who ran the infant orangutan.

tRequests for reprints should be sent to Donald Robbins, Department of Psychology, Emory University, Atlanta, Georgia 30322. these data are best understood in terms of the visual system's ability to process contours. The results of Dodwell et al (1970) are cited as evidence of the generality across species of the phenomenon, since they used squirrel monkeys (Samiri scuireus). A major difference in the squirrel monkey study was that, although both Ss showed the positive anomalous effect, the negative anomaly was found only when the Ss were tested on the positive effect first. Furthermore, both Ss were given two retraining series (to criterion), followed by the two types of transfer tests. Again Dodwell et al (1970) reported finding the negative effect only after the Ss had been tested on (and shown) the positive anomaly first. It is puzzling, as pointed out by Dodwell et al (1970), that the negative anomalous effect was found only after the positive anomaly was elicited. It is also puzzling that there appeared to be no transfer from one test phase to another. Dodwell et al gave their Ss all of the transfer tests in 2 days; all of one type on the first day of transfer and all of the other type on the second, and last, transfer test day. In the present study, a larger variety of transfer tests was administered over a 10-day period, each type of test being administered each day in an attempt to see if the Dodwell et al (1970) finding was due to the method of testing.

Dodwell (1970) criticized Krechevsky's interpretation of the effect as being inadequate today in view of our present knowledge of the mammalian visual system. Krechevsky (1938) had explained the effect in terms of Gestalt principles. If one views the stimuli in Fig. 1 as, for example, representations of a straight line, the solid lines may be considered to be the best representation of a straight line. If this is what is learned during training, then one might expect the positive transfer effect. Further, since the best representation of lines was avoided in the negative context, it would also explain the negative effect. As a result, Dodwell's rejection of Krechevsky's explanation may have been premature. In the present study, an additional stimulus was included, as shown in an example in Fig. 1C; the essential aspect is that it had fewer breaks if viewed as a solid line than the training stimuli.

In the present study, after performing the simultaneous discrimination shown in Fig. 1A to criterion, Ss were given a series of paired-comparison tests to assess this view as well as to test the generality of the anomalous transfer effects by using a testing procedure different from Dodwell et al (1970).

\section{METHOD \\ Subjects}

Two adult squirrel monkeys (Samiri scuireus) that had had extensive experience in learning sets completed the study. 

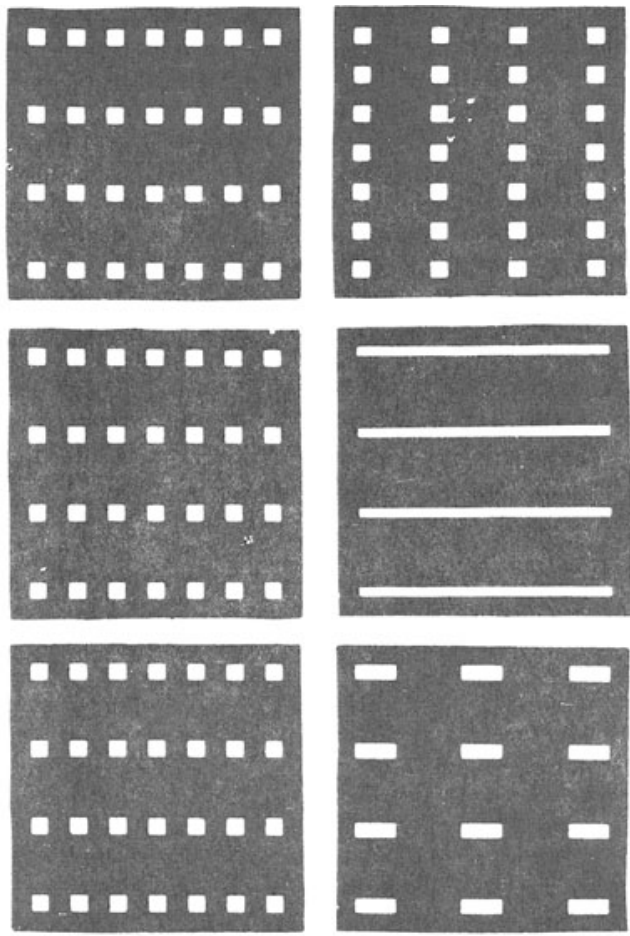

Fig. 1. Panel A (top): The training stimuli-dashed lines in the horizontal (positive) and vertical (negative) orientation. Panel B (middle): Example of transfer test stimuli-the positive training and horizontal solid lines. Panel $\mathrm{C}$ (bottom): Example of the additional transfer test stimuli-the positive training and the horizontal three-dash lines.

\section{Apparatus and Stimuli}

The apparatus was a modified small-animal test apparatus. A wooden form board with two foodwells over which small stands were placed was used. The stimuli were mounted on the stands and measured $2 \times 2$ in. The total amount of white area was the same for all stimuli.

\section{Procedure}

After shaping with three-dimensional objects, the Ss were given training on the simultaneous discrimination problem shown in Fig. 1A. A noncorrection procedure was used. Correct responses were rewarded with a sugar pellet. The left-right order of the stimuli was determined by a quadruple alternation schedule which required four consecutive correct responses before the stimulus positions were reversed. After meeting criterion, Ss were switched to a quasirandom schedule of position and slowly "thinned" from $100 \%$ reward for correct responses to $50 \%$ reward for correct responses. This was followed by 10 days of transfer tests which were nonrewarded, with all 15 possible combinations of the six stimuli being given each S. Two trials of each type were given each day, interspersed among additional training trials. Four Ss began the study; one developed diarrhea and the other failed to respond during the shaping phase, leaving two Ss, both of whom had the horizontal orientation as the positive and the vertical orientation as the negative stimulus.

\section{RESULTS AND DISCUSSION}

Both Ss had 10 days of training on the quadruple schedule and then 22 and 23 days of 60 trials per day for Ami and Ghengis, respectively, on the quasirandom presentation schedule, after which they were both responding at at least $90 \%$ correct. This level was maintained throughout the testing phase.

The results of the transfer tests are shown in Table 1 and are presented in terms of the number of times (out of 20) the row stimulus was preferred over the column stimulus for both Ami (the top values) and Ghengis (the lower values). These data represent the two trials of each type of transfer test over the 10-day testing phase, resulting in 20 trials on each of the 15 possible combinations of the six stimuli.

The results in the first row/first column represent nonrewarded training trials and reveal that the discrimination was maintained on rewarded and nonrewarded trials as well, since $95 \%$ and $85 \%$ correct responses were obtained for Ami and Ghengis, respectively. The positive transfer anomalous test is shown in the first row/second column and reveals a failure to obtain the positive anomaly, since the Ss showed a $95 \%$ and $90 \%$ preference for the original positive stimulus over the solid lines in the same orientation for Ami and Ghengis, respectively. The negative anomaly did appear (as shown in the second row/third column), in that a $75 \%$ and $80 \%$ preference for the original negative stimulus was found. Furthermore, the original positive stimulus was preferred over all of the stimuli tested (Row 1), a range of $85 \%-100 \%$ preference for the original positive stimulus. Excluding the positive stimulus, the negative stimulus was preferred to the other stimuli (Row 2), but the preference was not as strong as that found for the

Table 1

Number of Times (of 20) During the Transfer Test that the Row Stimulus Was Preferred Over the Column Stimulus for Ami (Top Values) and Ghengis (Lower Values)

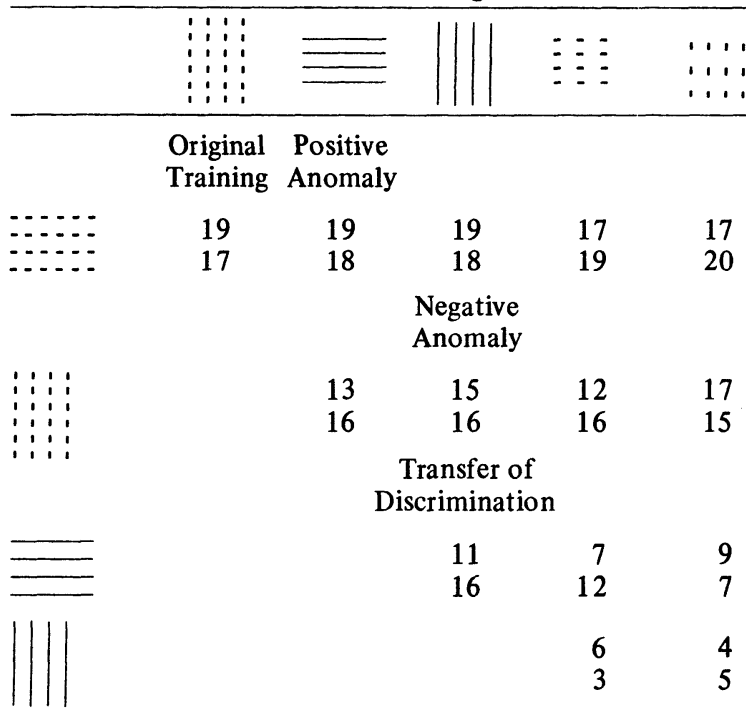

Transfer of Discrimination

$\because:-10$

$\because:-10$


positive stimulus, a range of $60 \%-85 \%$. When both stimuli had solid lines (third row/third column), there was a failure to find a substantial transfer of the discrimination, since the positive orientation preference was $55 \%$ and $80 \%$ for the two Ss. Similarly, for the three-dash horizontal stimuli (fifth row/fifth column) little preference (35\% and 50\%) was found. Finally, the solid positive orientation vs the nontraining stimuli revealed no preference, while the solid negative orientation was less preferred than the nontraining dashed stimuli.

In summary, these data reveal strong preferences for the original positive and negative stimuli; solid lines in the positive orientation revealed no preference, while solid lines in the negative orientation appeared to be avoided. In addition, little evidence of a transfer of the discrimination occurred. In the present study, transfer tests were carried out over a 10-day period. Dodwell et al (1970) gave only two types of transfer tests, and all of one type was given on a single day. In fact, their failure to find a negative effect except when it followed the testing (and appearance) of the positive effect implicates transfer testing procedures as a potentially important variable. Additional evidence in this regard comes from the results of a study with one 2-year-old orangutan (Pongo pygmelus). After performance on the simultaneous discrimination shown in Fig. $1 \mathrm{~A}$ achieved 95\% performance, 10 trials on each type of nonrewarded transfer test were inserted each day among additional training trials for 5 days. The results showed no transfer of the discrimination to solid lines on Day 1, followed by a $78 \%$ preference on Days $2-5$ for the solid-line stimulus in the positive orientation. In addition, the positive anomaly was found on Day 1 (75\% preference for solid over the original positive stimulus), although this preference was reversed on Days 2-5 with a $97 \%$ preference for the original positive stimulus. On Day 1 the negative anomaly was not found $(75 \%$ preference for the solid-line stimulus in the same orientation as the original negative stimulus), but on Days 2-5 the negative anomaly was found ( $84 \%$ preference). Thus, when testing is carried out over a number of days, preferences change over days. These data further support the contention that additional exploration of the various transfer test procedures must be pursued before we can be assured of the generality of the anomalous transfer effects.

\section{REFERENCES}

Dodwell, P. C. Anomalous transfer effects after pattern discrimination training in rats and squirrels. Journal of Comparative \& Physiological Psychology, 1970, 71, 42-51.

Dodwell, P. C., Litner, J. S., \& Niemi, R. R. Anomalous transfer effects following pattern discrimination training in the squirrel monkey. Psychonomic Science, 1970, 18, 5-7.

Krechevsky, I. An experimental investigation of the principle of proximity in the visual perception of the rat. Journal of Experimental Psychology, 1938, 22, 497-523.

(Received for publication November 6, 1972.) 